\title{
Topological Characterization, Measures of Uncertainty and Rough Equality of Sets on Two Universal Sets
}

\author{
D. P. Acharjya, B. K. Tripathy \\ School of Computing Science \& Engineering, VIT University, Vellore, TamilNadu, India \\ Email:dpacharjya@gmail.com; tripathybk@rediffmail.com
}

\begin{abstract}
The notion of rough set captures indiscernibility of elements in a set. But, in many real life situations, an information system establishes the relation between different universes. This gave the extension of rough set on single universal set to rough set on two universal sets. In this paper, we introduce rough equality of sets on two universal sets and rough inclusion of sets employing the notion of the lower and upper approximation. Also, we establish some basic properties that refer to our knowledge about the universes.
\end{abstract}

Index Terms - Rough Set, Solitary Set, Boolean Matrix, Rough Equality, Rough Inclusion

\section{Introduction}

In modern era of computing, there is a need of development in data analysis and knowledge representation. Many new mathematical modeling tools are emerging to the thrust of the real world task. Fuzzy set by Zadeh [1], rough set theory [2, 3], soft set by Molodtsov [4] are such mathematical models gained its popularity in past few decades. Development of these techniques and tools are studied under different domains like knowledge discovery in database, computational intelligence, knowledge engineering, granular computing etc. [5, 6, 7, 8, 9, 10].

The rough set $[2,3]$ philosophy specifies about the depth understanding of the object and its attributes influencing the object with a depicted value. So, there is a need to classify objects of the universe based on the indiscernibility relation between them. The basic idea of rough set is based upon the approximation of sets by pair of sets known as lower approximation and upper approximation. Here, the lower approximation and upper approximation operators are based on equivalence relation. However, the requirement of equivalence relation is a restrictive condition that may limit the application of rough set model. Therefore, rough set is generalized to some extent. For instance, the equivalence relation is generalized to binary relations $[11,12,13,14,15,16]$, neighborhood systems
[17], coverings [18], Boolean algebras [19, 20], fuzzy lattices [21], and completely distributive lattices [22].

On the other hand, rough set is generalized to fuzzy environment such as fuzzy rough set [23], and rough fuzzy set [24]. Further, the indiscernibility relation is generalized to almost indiscernibility relation to study many real life problems. The concept of rough set on fuzzy approximation spaces based on fuzzy proximity relation is studied by Acharjya and Tripathy [25, 26]. Further it is generalized to intuitionistic fuzzy proximity relation, and the concept of rough set on intuitionistic fuzzy approximation space is studied by Tripathy [27]. The different applications are also studied by the authors [28, 29, 30]. Further rough set of Pawlak is generalized to rough set on two universal sets with generalized approximation spaces and interval structure [31]. We continue a further study in the same direction.

The rest of the paper is organized as follows: Section 2 presents the foundations of rough set based on two universal sets and its topological characterization. In Section 3, we study the measures of uncertainty due to rough sets on two universal sets. Rough equality of sets on two universal sets and its properties are studied in Section 4. In Section 5, we introduce rough inclusion of sets on two universal sets. This is further followed by a conclusion in Section 6.

\section{Rough Set Based on Two Universal Sets}

An information system is a table that provides a convenient way to describe a finite set of objects called the universe by a finite set of attributes thereby representing all available information and knowledge. But, in many real life situations, an information system establishes the relation between different universes. This gave the extension of rough set on single universal set to rough set on two universal sets. Wong et. al [31] generalized the rough set models using two distinct but related universal sets. Let $U$ and $V$ be two universal sets and $R \subseteq(U \times V)$ be a binary relation. By a knowledge base, we understand the relational system $(U, V, R)$ an approximation space. For an element $x \in U$, we define the right neighborhood or the $R$-relative set of $x$ in $U$, 
$r(x)$ as $r(x)=\cup\{y \in V:(x, y) \in R\}$. Similarly for an element $y \in V$, we define the left neighborhood or the $R$ relative set of $y$ in $V, l(y)$ as $l(y)=\cup\{x \in U:(x, y) \in$ $\in R\}$

For any two elements $x_{1}, x_{2} \in U$, we say $x_{1}$ and $x_{2}$ are equivalent if $r\left(x_{1}\right)=r\left(x_{2}\right)$. Therefore, $\left(x_{1}, x_{2}\right) \in E_{U}$ if and only if $r\left(x_{1}\right)=r\left(x_{2}\right)$, where $E_{U}$ denote the equivalence relation on $U$. Hence, $E_{U}$ partitions the universal set $U$ into disjoint subsets. Similarly for any two elements $y_{1}, y_{2} \in V$, we say $y_{1}$ and $y_{2}$ are equivalent if $l\left(y_{1}\right)=l\left(y_{2}\right)$. Thus, $\left(y_{1}, y_{2}\right) \in E_{V}$ if and only if $l\left(y_{1}\right)=l\left(y_{2}\right)$, where $E_{V}$ denote the equivalence relation on $V$ and partitions the universal set $V$ into disjoint subsets. Therefore for the approximation space $(U, V, R)$, it is clear that $E_{V} \circ R=R=R \circ E_{U}$, where $E_{V} \circ R$ is the composition of $R$ and $E_{V}$.

For any $Y \subseteq V$ and the binary relation $R$, we associate two subsets $\underline{R} Y$ and $\bar{R} Y$ called the $R$-lower and $R$-upper approximations of $Y$ respectively, which are given by:

$$
\begin{aligned}
& \underline{R} Y=\cup\{x \in U: r(x) \subseteq Y\} \\
& \bar{R} Y=\cup\{x \in U: r(x) \cap Y \neq \phi\}
\end{aligned}
$$

The $R$-boundary of $Y$ is denoted as $B N_{R}(Y)$ and is given as $B N_{R}(Y)=\bar{R} Y-\underline{R} Y$. The pair $(\underline{R} Y, \bar{R} Y)$ is called as the rough set of $Y \subseteq V$ if $\underline{R} Y \neq \bar{R} Y$ or equivalently $B N_{R}(Y) \neq \phi$. Further, if $U$ and $V$ are finite sets, then the binary relation $R$ from $U$ to $V$ can be represented as $R(x, y)$, where

$$
R(x, y)= \begin{cases}1 & \text { if }(x, y) \in R \\ 0 & \text { if }(x, y) \notin R\end{cases}
$$

The characteristic function of $X \subseteq U$ is defined for each $x \in U$ as follows:

$$
X(x)= \begin{cases}1 & \text { if } x \in U \\ 0 & \text { if } x \notin U\end{cases}
$$

Therefore, the $R$-lower and $R$-upper approximations can be also presented in an equivalent form as shown below, where $\wedge$ and $\vee$ denotes the minimum and maximum operators respectively.

$$
\begin{aligned}
& (\underline{R Y}) x=\underset{y \in V}{\wedge}((1-R(x, y)) \vee Y(y)) \\
& (\bar{R} Y) x=\underset{y \in V}{\vee}(R(x, y) \wedge Y(y))
\end{aligned}
$$

Example 2.1 Let $U=\left\{x_{1}, x_{2}, x_{3}, x_{4}, x_{5}\right\}$ and $V=\left\{y_{1}\right.$, $\left.y_{2}, y_{3}, y_{4}, y_{5}, y_{6}\right\}$. Consider the relation $R$ given by its Boolean matrix:

$$
R=\left(\begin{array}{llllll}
1 & 1 & 0 & 0 & 1 & 0 \\
0 & 0 & 1 & 0 & 0 & 1 \\
0 & 1 & 0 & 1 & 0 & 0 \\
1 & 0 & 1 & 1 & 1 & 1 \\
1 & 1 & 0 & 0 & 1 & 0
\end{array}\right)
$$

From the above relation $R$ it is clear that, $r\left(x_{1}\right)=\left\{y_{1}, y_{2}, y_{5}\right\} ; r\left(x_{2}\right)=\left\{y_{3}, y_{6}\right\} ; \quad r\left(x_{3}\right)=\left\{y_{2}, y_{4}\right\} ;$ $r\left(x_{4}\right)=\left\{y_{1}, y_{3}, y_{4}, y_{5}, y_{6}\right\} \quad$ and $\quad r\left(x_{5}\right)=\left\{y_{1}, y_{2}, y_{5}\right\}$. Therefore, we get $U / E_{U}=\left\{\left\{x_{1}, x_{5}\right\},\left\{x_{2}\right\},\left\{x_{3}\right\},\left\{x_{4}\right\}\right\}$. Similarly, $V / E_{V}=\left\{\left\{y_{1}, y_{5}\right\},\left\{y_{3}, y_{6}\right\},\left\{y_{2}\right\},\left\{y_{4}\right\}\right\}$.Let us consider the target set $Y=\left\{y_{1}, y_{2}, y_{4}, y_{5}\right\}$. Therefore, the $R$-lower approximation, $\underline{R} Y$ is given as $\underline{R} Y=\left\{x_{1}, x_{3}, x_{5}\right\}$ whereas the $R$-upper approximation, $\bar{R} Y$ is given as $\bar{R} Y=\left\{x_{1}, x_{3}, x_{4}, x_{5}\right\}$. The $R$-boundary of $Y$ is given as $B N_{R}(Y)=\left\{x_{4}\right\}$.

Definition 2.1 Let $U$ and $V$ be two universal sets. Let $R$ be a binary relation from $U$ to $V$. If $x \in U$ and $r(x)=\phi$, then we call $x$ is a solitary element with respect to $R$. The set of all solitary elements with respect to the relation $R$ is called as solitary set and is denoted as $S$. Mathematically,

$$
S=\{x \in U: r(x)=\phi\}
$$

\subsection{Algebraic Properties of Rough Set based on Two Universal Sets}

In this section, we list the algebraic properties as established by Guilong Liu [32] that are interesting and valuable in the theory of rough sets as below. Let $R$ be an arbitrary binary relation from $U$ to $V$. Let $S$ be a solitary set with respect to the relation $R$. For subsets $X$, $Y$, in $V$

$$
\bar{R} Y=\underset{y \in Y}{\cup} l(y)
$$

(ii) $\underline{R} \phi=S, \bar{R} \phi=\phi, \underline{R} V=U$ and $\bar{R} V=S^{\prime}$, where $S^{\prime}$ denotes the complement of $S$ in $U$.

(iii) $S \subseteq \underline{R} X$ and $\bar{R} X \subseteq S^{\prime}$

(iv) $\quad \underline{R} X-S \subseteq \bar{R} X$

(v) $\quad \underline{R} X=U$ if and only if $\underset{x \in U}{\cup} r(x) \subseteq X ; \bar{R} X=\phi$ if and only if $X \subseteq\left(\cup_{x \in U} r(x)\right)^{\prime}$ 
(vi) If $S \neq \phi$, then $\underline{R} X \neq \bar{R} X$ for all $X \in P(V)$, where $P(V)$ denotes the power set of $V$.

(vii) For any given index set $I, X_{i} \in P(V)$, $\underline{R}\left(\cap_{i \in I} X_{i}\right)=\underset{i \in I}{\cap} \underline{R} X_{i}$ and $\bar{R}\left(\cup_{i \in I} X_{i}\right)=\cup_{i \in I}^{\cup} \bar{R} X_{i}$

(viii) If $X \subseteq Y$, then $\underline{R} X \subseteq \underline{R} Y$ and $\bar{R} X \subseteq \bar{R} Y$

(ix) $\quad \underline{R} X \cup \underline{R} Y \subseteq \underline{R}(X \cup Y)$, and

$$
\bar{R}(X \cap Y) \subseteq \bar{R} X \cap \bar{R} Y
$$

(x) $\quad(\underline{R} X)^{\prime}=\bar{R} X^{\prime}$, and $(\bar{R} X)^{\prime}=\underline{R} X^{\prime} ;$

(xi) There exists some $X \in P(U)$ such that $\underline{R} X=\bar{R} X$ if and only if $R$ is serial.

(xii) If $G$ is another binary relation from $U$ to $V$ and $\bar{R} X=\bar{G} X$ for all $x \in P(V)$, then $R=G$.

(xiii) If $G$ is another binary relation from $U$ to $V$ and $\underline{R} X=\underline{G} X$ for all $x \in P(V)$, then $R=G$.

\subsection{Topological Characterization of Rough Set based on Two Universal Sets}

In this section, we introduce an interesting topological characterization of rough set on two universal sets employing the notion of the lower and upper approximation. It results four important and different types of rough sets on two universal sets as discussed by Acharjya and Tripathy [33].

Type 1: If $\underline{R} Y \neq \phi$ and $\bar{R} Y \neq U$, then we say that $Y$ is roughly $R$-definable on two universal sets.

Type 2: If $\underline{R} Y=\phi$ and $\bar{R} Y \neq U$, then we say that $Y$ is internally $R$-undefinable on two universal sets.

Type 3: If $\underline{R} Y \neq \phi$ and $\bar{R} Y=U$, then we say that $Y$ is externally $R$-undefinable on two universal sets.

Type 4: If $\underline{R} Y=\phi$ and $\bar{R} Y=U$, then we say that $Y$ is totally $R$-undefinable on two universal sets.

\subsection{Table of Union}

In this section, we discuss the set theoretic operations such as union on types of rough sets on two universal sets. We state the corresponding tables for the set theoretic operation union. From the Table 1, it is clear that seven cases consist of ambiguous. In one case it can be any one of the four types. These ambiguities are due to inclusion $\underline{R} X \cup \underline{R Y} \subseteq \underline{R}(X \cup Y)$. The necessary proofs of ambiguity cases are thoroughly studied by Acharjya and Tripathy [33].

Table 1: Table of union

\begin{tabular}{|l|l|l|l|l|}
\hline U & \multicolumn{1}{|c|}{ Type 1 } & \multicolumn{1}{|c|}{ Type 2 } & \multicolumn{1}{|c|}{ Type 3 } & \multicolumn{1}{|c|}{ Type 4 } \\
\hline Type 1 & Type 1 / Type 3 & Type 1 / Type 3 & Type 3 & Type 3 \\
\hline Type 2 & Type 1 / Type 3 & Type 1 / Type 2 / Type 3 / Type 4 & Type 3 & Type 3 / Type 4 \\
\hline Type 3 & Type 3 & Type 3 & Type 3 & Type 3 \\
\hline Type 4 & Type 3 & Type 3 / Type 4 & Type 3 & Type 3 / Type 4 \\
\hline
\end{tabular}

\subsection{Table of Intersection}

Unlike union operation, it is interesting to see from the Table 2 that, out of sixteen cases for intersection, seven cases are ambiguous. Also it is observed that, in one case it can be any one of the four types. These ambiguities are due to inclusion $\bar{R}(X \cap Y) \subseteq \bar{R} X \cap \bar{R} Y$. The necessary proofs of ambiguity cases are thoroughly studied by Acharjya and Tripathy [33].

Table 2: Table of intersection

\begin{tabular}{|l|l|l|l|l|}
\hline$\bigcap$ & \multicolumn{1}{|c|}{ Type 1 } & \multicolumn{1}{|c|}{ Type 2 } & \multicolumn{1}{c|}{ Type 3 } & \multicolumn{1}{c|}{ Type 4 } \\
\hline Type 1 & Type 1 / Type 2 & Type 2 & Type 1 / Type 2 & Type 2 \\
\hline Type 2 & Type 2 & Type 2 & Type 2 & Type 2 \\
\hline Type 3 & Type 1 / Type 2 & Type 2 & Type 1 / Type 2 / Type 3 / Type 4 & Type 2 / Type 4 \\
\hline Type 4 & Type 2 & Type 2 & Type 2 / Type 4 & Type 2 / Type 4 \\
\hline
\end{tabular}

\section{Measures of Uncertainty}

The rough set $[2,3]$ philosophy specifies about the understanding of the objects and their attributes influencing the objects with a depicted value. So, there is a need to classify objects of the universe based on the indiscernibility relation between them. The basic idea of rough set is based upon the approximation of sets by a 
pair of sets known as the lower approximation and upper approximation of the set. Here, the lower and upper approximation operators are based on equivalence relation. However, the requirement of equivalence relation is a restrictive condition that may limit the application of rough set model. Therefore, rough set is generalized by Guilong Liu [32] to rough set on two universal sets. Because we are interested in classifications based on binary relation, it is interesting to have the idea of approximation of classifications. It is because classifications of universes play central roles in rough set theory. Recently Acharjya and Tripathy [34] have established important results and measures of uncertainty such as accuracy and quality of approximation employing the binary relation $R$ and discuss on properties of classifications. However, for completeness of the paper, we state the basic definitions and notions of measures of uncertainty.

Definition 3.1 Let $F=\left\{Y_{1}, Y_{2}, \cdots, Y_{n}\right\}$, where $n>1$ be a family of non empty sets defined over $V$. We say that $F$ is a classification of $V$ if and only if $\left(Y_{i} \cap Y_{j}\right)=\phi$ for $i \neq j$ and $\sum_{k=1}^{n} Y_{k}=V$.

Definition 3.2 Let $F=\left\{Y_{1}, Y_{2}, \cdots, Y_{n}\right\}$ be a family of non empty classification of $V$ and let $R$ be a binary relation from $U \rightarrow V$. Then the $R$-lower and $R$-upper approximation of the family $F$ is given as $\underline{R} F=\left\{\underline{R} Y_{1}, \underline{R} Y_{2}, \cdots, \underline{R} Y_{n}\right\} \quad$ and $\quad \bar{R} F=\left\{\bar{R} Y_{1}, \bar{R} Y_{2}, \cdots, \bar{R} Y_{n}\right\}$ respectively.

Definition 3.3 The accuracy of approximation of $F$ that expresses the percentage of possible correct decisions when classifying objects employing the binary relation $R$ is defined as

$$
\alpha_{R}(F)=\frac{\sum \operatorname{card}\left(\underline{R} Y_{i}\right)}{\sum \operatorname{card}\left(\bar{R} Y_{i}\right)} \text { for } i=1,2,3, \cdots, n .
$$

Definition 3.4 The quality of approximation of $F$ that expresses the percentage of objects which can be correctly classified to classes of $F$ by the binary relation $R$ is defined as

$$
v_{R}(F)=\frac{\sum \operatorname{card}\left(\underline{R}_{i}\right)}{\operatorname{card}(V)} \text { for } i=1,2,3, \cdots, n
$$

Definition 3.5 We say that $F=\left\{Y_{1}, Y_{2}, \cdots, Y_{n}\right\}$ is $R$ definable if and only if $\underline{R} F=\bar{R} F$; that is $\underline{R} Y_{i}=\bar{R} Y_{i}$ for $i=1,2,3, \cdots, n$.

Theorem 3.1 Let $R$ be a binary relation from $U \rightarrow V$ and let $F=\left\{Y_{1}, Y_{2}, \cdots, Y_{n}\right\}$, where $n>1$ be a classification of $V$. For any $R$-definable classification $F$ in $U, \alpha_{R}(F)=v_{R}(F)=1$. Hence, if a classification $F$ is $R$-definable then it is totally independent on $R$.

Theorem 3.2 Let $R$ be a binary relation from $U \rightarrow V$ and let $F=\left\{Y_{1}, Y_{2}, \cdots, Y_{n}\right\}$, where $n>1$ be a classification of $V$. If $\alpha_{R}(F)=v_{R}(F)=1$, then $F$ is $R$ definable in $V$.

Theorem 3.3 Let $R$ be a binary relation from $U \rightarrow V$ and for any classification $F=\left\{Y_{1}, Y_{2}, \cdots, Y_{n}\right\}$, $n>1$ in $V, 0 \leq \alpha_{R}(F) \leq v_{R}(F) \leq 1$.

\section{Rough Equality of Sets on Two Universal Sets}

The concept of rough set differs essentially from the ordinary concept of the set in that for the rough sets we are unable to define uniquely the membership relation. In set theory, two sets are said to be equal if they have same elements. However it is not true in case of rough sets. Therefore, the concept of rough (approximate) equality is introduced by Novotny and Pawlak [35]. Thus two sets can be unequal in set theory, but can be approximately equal. This is an important feature and according to our state of knowledge, the sets have close features which are enough to be assumed approximately equal. This is due to the indiscernibility relation between the objects of the universe. But, the indiscernibility relation is a restrictive relation that may limit the application of rough set. Therefore, rough set has extended to the settings of rough set on two universal sets based on binary relation. Hence the above concept of rough equality of sets can be extended to the settings of rough equality of sets on two universal sets. In fact we introduce three kinds of rough equality of sets on two universal sets. Now we present the formal definitions.

Definition 4.1 Let $U$ and $V$ be two universal sets and $R \subseteq(U \times V)$ be a binary relation. Let the relational system $(U, V, R)$ be a knowledge base, and $Y_{1}, Y_{2} \subseteq V$. We say that

(i) Sets $Y_{1}$ and $Y_{2}$ are bottom $R$-equal in $V$ if $\underline{R} Y_{1}=\underline{R} Y_{2}$. We write it as $Y_{1} \approx_{B} Y_{2}$.

(ii) Sets $Y_{1}$ and $Y_{2}$ are top $R$-equal in $V$ if $\bar{R} Y_{1}=\bar{R} Y_{2}$. We write it as $Y_{1} \approx_{T} Y_{2}$.

(iii) Sets $Y_{1}$ and $Y_{2}$ are $R$-equal in $V$ if $Y_{1} \approx_{B} Y_{2}$ and $Y_{1} \approx_{T} Y_{2}$. We write it as $Y_{1} \approx Y_{2}$.

We associate the following physical interpretations, with the above notion of rough equality of sets on two universal sets. If $Y_{1} \approx_{B} Y_{2}$, this means that positive 
examples of the sets $Y_{1}$ and $Y_{2}$ in $V$ are equal. If $Y_{1} \approx_{T} Y_{2}$, then the negative examples of the sets $Y_{1}$ and $Y_{2}$ in $V$ are equal. If $Y_{1} \approx Y_{2}$, this means that both positive and negative examples of the sets $Y_{1}$ and $Y_{2}$ in $V$ are the same.

Example 4.1 Let $U=\left\{x_{1}, x_{2}, x_{3}, x_{4}, x_{5}\right\}$ and $V=$ $\left\{y_{1}, y_{2}, y_{3}, y_{4}, y_{5}, y_{6}, y_{7}, y_{8}\right\}$. Consider the binary relation $R$ as $R=\left\{\left(x_{1}, y_{2}\right),\left(x_{1}, y_{3}\right),\left(x_{2}, y_{1}\right),\left(x_{2}, y_{4}\right)\right.$, $\left(x_{2}, y_{5}\right),\left(x_{3}, y_{3}\right),\left(x_{3}, y_{6}\right),\left(x_{3}, y_{7}\right),\left(x_{4}, y_{1}\right),\left(x_{4}, y_{7}\right)$, $\left.\left(x_{4}, y_{8}\right),\left(x_{5}, y_{2}\right),\left(x_{5}, y_{3}\right)\right\}$. Thus $R$ can be written in its Boolean matrix form as:

$$
R=\left(\begin{array}{llllllll}
0 & 1 & 1 & 0 & 0 & 0 & 0 & 0 \\
1 & 0 & 0 & 1 & 1 & 0 & 0 & 0 \\
0 & 0 & 1 & 0 & 0 & 1 & 1 & 0 \\
1 & 0 & 0 & 0 & 0 & 0 & 1 & 1 \\
0 & 1 & 1 & 0 & 0 & 0 & 0 & 0
\end{array}\right)
$$

From the above relation $R$ it is clear that, $r\left(x_{1}\right)=$ $\left\{y_{2}, y_{3}\right\} ; r\left(x_{2}\right)=\left\{y_{1}, y_{4}, y_{5}\right\} ; r\left(x_{3}\right)=\left\{y_{3}, y_{6}, y_{7}\right\} ; r\left(x_{4}\right)$ $=\left\{y_{1}, y_{4}, y_{5}\right\} ;$ and $r\left(x_{5}\right)=\left\{y_{2}, y_{3}\right\}$. Therefore, we get $U \mid E_{U}=\left\{\left\{x_{1}, x_{5}\right\},\left\{x_{2}\right\},\left\{x_{3}\right\},\left\{x_{4}\right\}\right\}$.

For sets $Y_{1}=\left\{y_{1}, y_{2}, y_{3}\right\}$ and $Y_{2}=\left\{y_{2}, y_{3}, y_{7}\right\}$ we have $\underline{R} Y_{1}=\left\{x_{1}, x_{5}\right\}=\underline{R} Y_{2}$, therefore $Y_{1} \approx_{B} Y_{2}$. Hence $Y_{1}$ and $Y_{2}$ are bottom $R$-equal in $V$. Again, on considering $Y_{1}=\left\{y_{1}, y_{2}, y_{7}\right\}$ and $Y_{2}=\left\{y_{2}, y_{3}, y_{4}, y_{8}\right\}$ we have $\bar{R} Y_{1}=$ $\left\{x_{1}, x_{2}, x_{3}, x_{4}, x_{5}\right\}=\bar{R} Y_{2}$, therefore $Y_{1} \approx_{T} Y_{2}$. Hence $Y_{1}$ and $Y_{2}$ are top $R$-equal in $V$. Similarly on taking $Y_{1}=$ $\left\{y_{2}, y_{4}, y_{6}\right\}$ and $Y_{2}=\left\{y_{3}, y_{4}, y_{6}\right\}$ we have $\underline{R} Y_{1}=\phi=\underline{R} Y_{2}$, and $\bar{R} Y_{1}=\left\{x_{1}, x_{2}, x_{3}, x_{5}\right\}=\bar{R} Y_{2}$, therefore $Y_{1} \approx Y_{2}$. Hence $Y_{1}$ and $Y_{2}$ are $R$-equal in $V$.

Proposition 4.1 The following properties of relations $\approx_{B}, \approx_{T}$, and $\approx$ are immediate consequences of the definitions. Let $U$ and $V$ be two universal sets and $R \subseteq$ $(U \times V)$ be a binary relation. Then for $Y_{1}, Y_{2} \subseteq V$, the following properties holds.

(a) $Y_{1} \approx_{B} Y_{2}$ if and only if $\left(Y_{1} \cap Y_{2}\right) \approx_{B} Y_{1}$ and $\left(Y_{1} \cap Y_{2}\right) \approx_{B} Y_{2}$.

(b) $Y_{1} \approx_{T} Y_{2}$ if and only if $\left(Y_{1} \cup Y_{2}\right) \approx_{T} Y_{1}$ and $\left(Y_{1} \cup Y_{2}\right) \approx_{T} Y_{2}$. (c) If $Y_{1} \approx_{T} Y_{1}^{\prime}$ and $Y_{2} \approx_{T} Y_{2}^{\prime}$, then $\left(Y_{1} \cup Y_{2}\right) \approx_{T}$ $\left(Y_{1}^{\prime} \cup Y_{2}^{\prime}\right)$

(d) If $Y_{1} \approx_{B} Y_{1}^{\prime}$ and $Y_{2} \approx_{B} Y_{2}^{\prime}$, then $\left(Y_{1} \cap Y_{2}\right) \approx_{B}$ $\left(Y_{1}^{\prime} \cap Y_{2}^{\prime}\right)$

(e) If $Y_{1} \subseteq Y_{2}$ and $Y_{2} \approx_{T} \phi$, then $Y_{1} \approx_{T} \phi$.

(f) If $Y_{1} \subseteq Y_{2}$ and $Y_{1} \approx_{T} V$, then $Y_{2} \approx_{T} V$.

(g) If $Y_{1} \approx_{B} \phi$ or $Y_{2} \approx_{B} \phi$, then $\left(Y_{1} \cap Y_{2}\right) \approx_{B} \phi$.

(h) If $Y_{1} \approx_{T} V$ or $Y_{2} \approx_{T} V$, then $\left(Y_{1} \cup Y_{2}\right) \approx_{T} V$.

Proof (a) Assume that $\left(Y_{1} \cap Y_{2}\right) \approx_{B} Y_{1}$ and $\left(Y_{1} \cap Y_{2}\right) \approx_{B}$ $Y_{2}$. It implies that $\underline{R}\left(Y_{1} \cap Y_{2}\right)=\underline{R}\left(Y_{1}\right)$ and $\underline{R}\left(Y_{1} \cap Y_{2}\right)=$ $\underline{R}\left(Y_{2}\right)$. Therefore, $\underline{R}\left(Y_{1}\right)=\underline{R}\left(Y_{2}\right)$ and hence $Y_{1} \approx_{B} Y_{2}$. Conversely assume that $Y_{1} \approx_{B} Y_{2}$. It implies that $\underline{R}\left(Y_{1}\right)$ $=\underline{R}\left(Y_{2}\right)$. But, $\underline{R}\left(Y_{1} \cap Y_{2}\right)=\underline{R}\left(Y_{1}\right) \cap \underline{R}\left(Y_{2}\right)=\underline{R}\left(Y_{1}\right)$ and $\underline{R}\left(Y_{1} \cap Y_{2}\right)=\underline{R}\left(Y_{1}\right) \cap \underline{R}\left(Y_{2}\right)=\underline{R}\left(Y_{2}\right)$. Therefore, we have $\left(Y_{1} \cap Y_{2}\right) \approx_{B} Y_{1}$ and $\left(Y_{1} \cap Y_{2}\right) \approx_{B} Y_{2}$.

Proof $(b)$ Assume that $\left(Y_{1} \cup Y_{2}\right) \approx_{T} Y_{1}$ and $\left(Y_{1} \cup Y_{2}\right) \approx_{T}$ $Y_{2}$. It implies that $\bar{R}\left(Y_{1} \cup Y_{2}\right)=\bar{R}\left(Y_{1}\right)$ and $\bar{R}\left(Y_{1} \cup Y_{2}\right)=$ $\bar{R}\left(Y_{2}\right)$. Therefore, $\bar{R}\left(Y_{1}\right)=\bar{R}\left(Y_{2}\right)$ and hence $Y_{1} \approx_{T} Y_{2}$. Conversely assume that $Y_{1} \approx_{T} Y_{2}$. It implies that $\bar{R}\left(Y_{1}\right)$ $=\bar{R}\left(Y_{2}\right)$. But, $\bar{R}\left(Y_{1} \cup Y_{2}\right)=\bar{R}\left(Y_{1}\right) \cup \bar{R}\left(Y_{2}\right)=\bar{R}\left(Y_{1}\right)$ and $\bar{R}\left(Y_{1} \cup Y_{2}\right)=\bar{R}\left(Y_{1}\right) \cup \bar{R}\left(Y_{2}\right)=\bar{R}\left(Y_{2}\right)$. Therefore, we have $\left(Y_{1} \cup Y_{2}\right) \approx_{T} Y_{1}$ and $\left(Y_{1} \cup Y_{2}\right) \approx_{T} Y_{2}$.

Proof $(c)$ Assume that $Y_{1} \approx_{T} Y_{1}^{\prime}$ and $Y_{2} \approx_{T} Y_{2}^{\prime}$. It implies that $\bar{R} Y_{1}=\bar{R} Y_{1}^{\prime}$ and $\bar{R} Y_{2}=\bar{R} Y_{2}^{\prime}$. But, $\bar{R}\left(Y_{1} \cup Y_{2}\right)=\bar{R} Y_{1}$ $\cup \bar{R} Y_{2}=\bar{R} Y_{1}^{\prime} \cup \bar{R} Y_{2}^{\prime}=\bar{R}\left(Y_{1}^{\prime} \cup Y_{2}^{\prime}\right)$. Therefore, $\left(Y_{1} \cup Y_{2}\right)$ $\approx_{T}\left(Y_{1}^{\prime} \cup Y_{2}^{\prime}\right)$

Proof $(d)$ Assume that $Y_{1} \approx_{B} Y_{1}^{\prime}$ and $Y_{2} \approx_{B} Y_{2}^{\prime}$. Thus we have $\underline{R} Y_{1}=\underline{R} Y_{1}^{\prime}$ and $\underline{R} Y_{2}=\underline{R} Y_{2}^{\prime}$. But, $\underline{R}\left(Y_{1} \cap Y_{2}\right)=\underline{R} Y_{1}$ $\cap \underline{R} Y_{2}=\underline{R} Y_{1}^{\prime} \cap \underline{R} Y_{2}^{\prime}=\underline{R}\left(Y_{1}^{\prime} \cap Y_{2}^{\prime}\right)$. Therefore, $\left(Y_{1} \cap Y_{2}\right)$ $\approx_{B}\left(Y_{1}^{\prime} \cap Y_{2}^{\prime}\right)$.

Proof (e) Assume that $Y_{1} \subseteq Y_{2}$ and $Y_{2} \approx_{T} \phi$. It implies that $\bar{R} Y_{1} \subseteq \bar{R} Y_{2}$ and $\bar{R} Y_{2}=\bar{R} \phi$. Therefore, $\bar{R} Y_{1} \subseteq \bar{R} Y_{2}=$ 
$\bar{R} \phi=\phi$, i.e., $\bar{R} Y_{1} \subseteq \phi$. So definitely, $\bar{R} Y_{1}=\phi=\bar{R} \phi$. Hence, $Y_{1} \approx_{T} \phi$.

Proof $(f)$ Assume that $Y_{1} \subseteq Y_{2}$ and $Y_{1} \approx_{T} V$. It implies that $\bar{R} Y_{1} \subseteq \bar{R} Y_{2}$ and $\bar{R} Y_{1}=\bar{R} V=S^{\prime}$. But $\bar{R} Y_{2} \supseteq \bar{R} Y_{1}=S^{\prime}$, i.e., $\bar{R} Y_{2} \supseteq S^{\prime}$. Since $S^{\prime}$ is the complement of the solitary set, definitely $\bar{R} Y_{2}=S^{\prime}$. Therefore, $\bar{R} Y_{2}=S^{\prime}=$ $\bar{R} V$. It implies that $Y_{2} \approx_{T} V$.

Proof $(g)$ Let us assume $Y_{1} \approx_{B} \phi$. It implies that $\underline{R} Y_{1}=$ $\underline{R} \phi=S$. Therefore, $\underline{R}\left(Y_{1} \cap Y_{2}\right)=\underline{R}_{1} \cap \underline{R}_{2}=S \cap \underline{R} Y_{2}$.

Since $S$ is a solitary set, definitely $S \cap \underline{R} Y_{2}$ is equal to $S$. Thus, $\underline{R}\left(Y_{1} \cap Y_{2}\right)=S=\underline{R} \phi$, i.e., $\underline{R}\left(Y_{1} \cap Y_{2}\right)=\underline{R} \phi$. Hence, $\left(Y_{1} \cap Y_{2}\right) \approx_{B} \phi$. Similarly it can be verified that if $Y_{2} \approx_{B} \phi$, then $\left(Y_{1} \cap Y_{2}\right) \approx_{B} \phi$.

Proof $(h)$ Let us assume $Y_{1} \approx_{T} V$. It implies that $\bar{R} Y_{1}=$ $\bar{R} V=S^{\prime}$. But, $\bar{R}\left(Y_{1} \cup Y_{2}\right)=\bar{R} Y_{1} \cup \bar{R} Y_{2}=S^{\prime} \cup \bar{R} Y_{2}=S^{\prime}=$ $\bar{R} V$. It indicates that, $\left(Y_{1} \cup Y_{2}\right) \approx_{T} V$. Similarly it can be verified that if $Y_{2} \approx_{T} V$, then $\left(Y_{1} \cup Y_{2}\right) \approx_{T} V$.

\section{Rough Inclusion of Sets on Two Universal Sets}

Inclusion relation is one of the fundamental concepts in set theory. An analogous notion in rough set is introduced by Pawlak [2]. Hence, rough inclusion of sets can be extended to the settings of rough inclusion of sets on two universal sets. We define the rough inclusion of sets on two universal sets in the same way as rough equality of sets on two universal sets. The formal definition of rough inclusion of sets on two universal sets is as follows.

Definition 5.1 Let $U$ and $V$ be two universal sets and $R \subseteq(U \times V)$ be a binary relation. Let the relational system $(U, V, R)$ be a knowledge base, and $Y_{1}, Y_{2} \subseteq V$. We say that

(i) Set $Y_{1}$ is bottom $R$-included in $Y_{2}$ if and only if $\underline{R} Y_{1} \subseteq \underline{R} Y_{2}$. We denote it as $Y_{1} \square_{B} Y_{2}$.

(ii) Set $Y_{1}$ is top $R$-included in $Y_{2}$ if and only if $\bar{R} Y_{1} \subseteq \bar{R} Y_{2}$. We denote it as $Y_{1} \square_{T} Y_{2}$.

(iii) Set $Y_{1}$ is said to be $R$-included in $Y_{2}$ if and only if $Y_{1} \square_{B} Y_{2}$ and $Y_{1} \square_{T} Y_{2}$. We denote it as $Y_{1} \square Y_{2}$.
Example 5.1 Let us consider the knowledge base as in Example 4.1. In this knowledge base for sets $Y_{1}=$ $\left\{y_{2}, y_{3}, y_{4}\right\}$ and $Y_{2}=\left\{y_{2}, y_{3}, y_{6}, y_{7}\right\}$ we have $R Y_{1}=\left\{x_{1}\right.$, $\left.x_{5}\right\}$ and $\underline{R} Y_{2}=\left\{x_{1}, x_{3}, x_{5}\right\}$. Therefore, $\underline{R} Y_{1} \subseteq \underline{R} Y_{2}$. It implies that $Y_{1}$ is bottom $R$-included in the set $Y_{2}$. Again on taking $Y_{1}=\left\{y_{2}, y_{3}, y_{6}\right\}$ and $Y_{2}=\left\{y_{2}, y_{7}\right\}$ we have $\bar{R} Y_{1}=\left\{x_{1}, x_{3}, x_{5}\right\}$ and $\bar{R} Y_{2}=\left\{x_{1}, x_{3}, x_{4}, x_{5}\right\}$. Thus, we get $\bar{R} Y_{1} \subseteq \bar{R} Y_{2}$. It indicates that $Y_{1}$ is top $R$-included in the set $Y_{2}$. Similarly on taking $Y_{1}=\left\{y_{2}, y_{3}\right\}$ and $Y_{2}=$ $\left\{y_{2}, y_{3}, y_{6}, y_{7}\right\}$ we have $\underline{R} Y_{1}=\left\{x_{1}, x_{5}\right\} ; \quad \underline{R} Y_{2}=\left\{x_{1}, x_{3}\right.$, $\left.x_{5}\right\} ; \bar{R} Y_{1}=\left\{x_{1}, x_{5}\right\}$ and $\bar{R} Y_{2}=\left\{x_{1}, x_{3}, x_{4}, x_{5}\right\}$. Therefore, we get $\underline{R} Y_{1} \subseteq \underline{R} Y_{2}$ and $\bar{R} Y_{1} \subseteq \bar{R} Y_{2}$. It indicates that $Y_{1}$ is $R$-included in the set $Y_{2}$.

Proposition 5.1 The following properties of relations $\square_{B}, \square_{T}$, and $\square$ are immediate consequences of the definitions. Let $U$ and $V$ be two universal sets and $R \subseteq$ $(U \times V)$ be a binary relation. Then for $Y_{1}, Y_{2} \subseteq V$, the following properties holds.

(i) If $Y_{1} \subseteq Y_{2}$, then $Y_{1} \square_{B} Y_{2}, Y_{1} \square_{T} Y_{2}$, and $Y_{1} \square Y_{2}$.

(j) $Y_{1} \square_{B} Y_{2}$ and $Y_{2} \square_{B} Y_{1}$, then $Y_{1} \approx_{B} Y_{2}$.

(k) $Y_{1} \square_{T} Y_{2}$ and $Y_{2} \square_{T} Y_{1}$, then $Y_{1} \approx_{T} Y_{2}$.

(l) If $Y_{1} \square Y_{2}$ and $Y_{2} \square Y_{1}$, then $Y_{1} \approx Y_{2}$.

(m) $\quad Y_{1} \square_{T} Y_{2}$ if and only if $\left(Y_{1} \cup Y_{2}\right) \approx_{T} Y_{2}$.

(n) $Y_{1} \square_{B} Y_{2}$ if and only if $\left(Y_{1} \cap Y_{2}\right) \approx_{B} Y_{1}$.

(o) If $Y_{1} \subseteq Y_{2}, Y_{1} \approx_{B} Y_{1}^{\prime}$ and $Y_{2} \approx_{B} Y_{2}^{\prime}$, then $Y_{1}^{\prime} \square_{B} Y_{2}^{\prime}$.

(p) If $Y_{1} \subseteq Y_{2}, Y_{1} \approx_{T} Y_{1}^{\prime}$ and $Y_{2} \approx_{T} Y_{2}^{\prime}$, then $Y_{1}^{\prime} \square_{T} Y_{2}^{\prime}$.

(q) If $Y_{1} \subseteq Y_{2}, Y_{1} \approx Y_{1}^{\prime}$ and $Y_{2} \approx Y_{2}^{\prime}$, then $Y_{1}^{\prime} \square Y_{2}^{\prime}$.

(r) If $Y_{1}^{\prime} \square_{T} Y_{1}$ and $Y_{2}^{\prime} \square_{T} Y_{2}$, then $\left(Y_{1}^{\prime} \cup Y_{2}^{\prime}\right) \square_{T}$ $\left(Y_{1} \cup Y_{2}\right)$.

(s) If $Y_{1}^{\prime} \square_{B} Y_{1}$ and $Y_{2}^{\prime} \square_{B} Y_{2}$, then $\left(Y_{1}^{\prime} \cap Y_{2}^{\prime}\right) \square_{B}$ $\left(Y_{1} \cap Y_{2}\right)$.

(t) $\quad\left(Y_{1} \cap Y_{2}\right) \square_{B} Y_{1} \square_{T}\left(Y_{1} \cup Y_{2}\right)$.

(u) If $Y_{1} \square_{B} Y_{2}$ and $Y_{1} \approx_{B} Y_{3}$, then $Y_{3} \square_{B} Y_{2}$. 
(v) If $Y_{1} \square_{T} Y_{2}$ and $Y_{1} \approx_{T} Y_{3}$, then $Y_{3} \square_{T} Y_{2}$.

(w) If $Y_{1} \square Y_{2}$ and $Y_{1} \approx Y_{3}$, then $Y_{3} \square Y_{2}$.

Proof (a) Assume that $Y_{1} \subseteq Y_{2}$. It implies that $\underline{R} Y_{1} \subseteq \underline{R} Y_{2}$ and $\bar{R} Y_{1} \subseteq \bar{R} Y_{2}$. Therefore, $Y_{1} \square_{B} Y_{2} ; Y_{1} \square_{T} Y_{2}$ and thus $Y_{1} \square Y_{2}$.

Proof (b) Suppose that $Y_{1} \square_{B} Y_{2}$ and $Y_{2} \square_{B} Y_{1}$. It implies that $\underline{R} Y_{1} \subseteq \underline{R}_{2}$ and $\underline{R} Y_{2} \subseteq \underline{R}_{1}$. Therefore, $\underline{R} Y_{1}=\underline{R}_{2}$. It indicates that $Y_{1} \approx_{B} Y_{2}$.

Proof (c) Suppose that $Y_{1} \square_{T} Y_{2}$ and $Y_{2} \square_{T} Y_{1}$. It implies that $\bar{R} Y_{1} \subseteq \bar{R} Y_{2}$ and $\bar{R} Y_{2} \subseteq \bar{R} Y_{1}$. Therefore, $\bar{R} Y_{1}=\bar{R} Y_{2}$. It indicates that $Y_{1} \approx_{T} Y_{2}$.

Proof (d) Suppose that $Y_{1} \square Y_{2}$ and $Y_{2} \square Y_{1}$. It implies that $Y_{1} \square_{B} Y_{2} ; Y_{1} \square_{T} Y_{2} ; Y_{2} \square_{B} Y_{1}$ and $Y_{2} \square_{T} Y_{1}$. Therefore, by above property $(b)$ and $(c)$ we get $Y_{1} \approx_{B} Y_{2}$ and $Y_{1} \approx_{T} Y_{2}$. Hence, we have $Y_{1} \approx Y_{2}$.

Proof (e) Assume that $Y_{1} \square_{T} Y_{2}$. It implies that $\bar{R} Y_{1} \subseteq \bar{R} Y_{2}$. But, $\bar{R}\left(Y_{1} \cup Y_{2}\right)=\bar{R} Y_{1} \cup \bar{R} Y_{2}=\bar{R} Y_{2}$. Thus we get, $\left(Y_{1} \cup Y_{2}\right) \approx_{T} Y_{2}$. Conversely, suppose that $\left(Y_{1} \cup Y_{2}\right)$ $\approx_{T} Y_{2}$. It implies that $\bar{R}\left(Y_{1} \cup Y_{2}\right)=\bar{R} Y_{2}$, i.e., $\bar{R} Y_{1} \cup \bar{R} Y_{2}$ $=\bar{R} Y_{2}$. It is possible only when $\bar{R} Y_{1} \subseteq \bar{R} Y_{2}$ or $\bar{R} Y_{1}=\phi$. Therefore, in either case $Y_{1} \square_{T} Y_{2}$.

Proof (f) Assume that $Y_{1} \square_{B} Y_{2}$. It implies that $\underline{R} Y_{1} \subseteq \underline{R} \underline{Y}_{2}$. But, $\underline{R}\left(Y_{1} \cap Y_{2}\right)=\underline{R} Y_{1} \cap \underline{R} Y_{2}=\underline{R} Y_{1}$. Thus we get, $\left(Y_{1} \cap Y_{2}\right) \approx_{B} Y_{1}$. Conversely, suppose that $\left(Y_{1} \cap Y_{2}\right)$ $\approx_{B} Y_{1}$. It implies that $\underline{R}\left(Y_{1} \cap Y_{2}\right)=\underline{R} Y_{1}$, i.e., $\underline{R} Y_{1} \cap \underline{R} Y_{2}$ $=\underline{R Y}_{1}$. It is possible only when $\underline{R} Y_{1} \subseteq \underline{R} Y_{2}$. Therefore, in either case $Y_{1} \square_{B} Y_{2}$.

Proof $(g)$ Assume that $Y_{1} \subseteq Y_{2} ; Y_{1} \approx_{B} Y_{1}^{\prime}$ and $Y_{2} \approx_{B} Y_{2}^{\prime}$. It implies that $\underline{R} Y_{1} \subseteq \underline{R} \underline{Y}_{2} ; \underline{R} Y_{1}=\underline{R} \underline{Y}_{1}^{\prime}$ and $\underline{R} Y_{2}=\underline{R} Y_{2}^{\prime}$. Therefore, $\underline{R} Y_{1}^{\prime} \subseteq \underline{R} Y_{2}^{\prime}$. It indicates that $Y_{1}^{\prime} \square_{B} Y_{2}^{\prime}$.

Proof $(h)$ Assume that $Y_{1} \subseteq Y_{2} ; Y_{1} \approx_{T} Y_{1}^{\prime}$ and $Y_{2} \approx_{T} Y_{2}^{\prime}$. It implies that $\bar{R} Y_{1} \subseteq \bar{R} Y_{2} ; \bar{R} Y_{1}=\bar{R} Y_{1}^{\prime}$ and $\bar{R} Y_{2}=\bar{R} Y_{2}^{\prime}$. Therefore, $\bar{R} Y_{1}^{\prime} \subseteq \bar{R} Y_{2}^{\prime}$. It indicates that $Y_{1}^{\prime} \square_{T} Y_{2}^{\prime}$.
Proof (i) Assume that $Y_{1} \subseteq Y_{2} ; Y_{1} \approx Y_{1}^{\prime}$ and $Y_{2} \approx Y_{2}^{\prime}$. It implies that $\bar{R} Y_{1} \subseteq \bar{R} Y_{2} ; \underline{R} Y_{1} \subseteq \underline{R} Y_{2} ; \underline{R} Y_{1}=\underline{R} Y_{1}^{\prime}$; $\bar{R} Y_{1}=\bar{R} Y_{1}^{\prime} ; \underline{R} Y_{2}=\underline{R} Y_{2}^{\prime}$ and $\bar{R} Y_{2}=\bar{R} Y_{2}^{\prime}$. Therefore, we have $\underline{R} Y_{1}^{\prime}=\underline{R} Y_{1} \subseteq \underline{R} Y_{2}=\underline{R} Y_{2}^{\prime}$, i.e., $\underline{R} Y_{1}^{\prime} \subseteq \underline{R} Y_{2}^{\prime}$. It indicates that $Y_{1}^{\prime} \square_{B} Y_{2}^{\prime}$. Similarly, $\bar{R} Y_{1}^{\prime}=\bar{R} Y_{1} \subseteq \bar{R} Y_{2}=$ $\bar{R} Y_{2}^{\prime}$, i.e., $\bar{R} Y_{1}^{\prime} \subseteq \bar{R} Y_{2}^{\prime}$. It indicates that $Y_{1}^{\prime} \square_{T} Y_{2}^{\prime}$. Thus on combining the results we get $Y_{1}^{\prime} \square Y_{2}^{\prime}$.

Proof (j) Suppose that $Y_{1}^{\prime} \square_{T} Y_{1}$ and $Y_{2}^{\prime} \square_{T} Y_{2}$. It implies that $\bar{R} Y_{1}^{\prime} \subseteq \bar{R} Y_{1}$ and $\bar{R} Y_{2}^{\prime} \subseteq \bar{R} Y_{2}$. But, $\bar{R}\left(Y_{1}^{\prime} \cup Y_{2}^{\prime}\right)=\bar{R} Y_{1}^{\prime}$ $\cup \bar{R} Y_{2}^{\prime} \subseteq \bar{R} Y_{1} \cup \bar{R} Y_{2}=\bar{R}\left(Y_{1} \cup Y_{2}\right)$. Thus, $\bar{R}\left(Y_{1}^{\prime} \cup Y_{2}^{\prime}\right)$ $\subseteq \bar{R}\left(Y_{1} \cup Y_{2}\right)$ and hence $\left(Y_{1}^{\prime} \cup Y_{2}^{\prime}\right) \square_{T}\left(Y_{1} \cup Y_{2}\right)$.

Proof (k) Suppose that $Y_{1}^{\prime} \square_{B} Y_{1}$ and $Y_{2}^{\prime} \square_{B} Y_{2}$. It implies that $\underline{R} Y_{1}^{\prime} \subseteq \underline{R} Y_{1}$ and $\underline{R} Y_{2}^{\prime} \subseteq \underline{R} Y_{2}$. But, $\underline{R}\left(Y_{1}^{\prime} \cap Y_{2}^{\prime}\right)=\underline{R Y}_{1}^{\prime}$ $\cap \underline{R}_{2}^{\prime} \subseteq \underline{R} \underline{Y}_{1} \cap \underline{R} \underline{Y}_{2}=\underline{R}\left(Y_{1} \cap Y_{2}\right)$. Thus, $\underline{R}\left(Y_{1}^{\prime} \cap Y_{2}^{\prime}\right)$ $\subseteq \underline{R}\left(Y_{1} \cap Y_{2}\right)$ and hence $\left(Y_{1}^{\prime} \cap Y_{2}^{\prime}\right) \square_{B}\left(Y_{1} \cap Y_{2}\right)$.

Proof $(l)$ We know that $\left(Y_{1} \cap Y_{2}\right) \subseteq Y_{1}$. Therefore, $\underline{R}\left(Y_{1} \cap Y_{2}\right) \subseteq \underline{R} Y_{1}$. It indicates that $\left(Y_{1} \cap Y_{2}\right) \square_{B} Y_{1}$. Similarly, $Y_{1} \subseteq\left(Y_{1} \cup Y_{2}\right)$. Therefore, $\bar{R} Y_{1} \subseteq \bar{R}\left(Y_{1} \cup Y_{2}\right)$ and hence $Y_{1} \square_{T}\left(Y_{1} \cup Y_{2}\right)$. On combining the results we get $\left(Y_{1} \cap Y_{2}\right) \square_{B} Y_{1} \square_{T}\left(Y_{1} \cup Y_{2}\right)$.

Proof $(m)$ Suppose that $Y_{1} \square_{B} Y_{2}$ and $Y_{1} \approx_{B} Y_{3}$. It implies that $\underline{R} Y_{1} \subseteq \underline{R} \underline{Y}_{2}$ and $\underline{R} Y_{1}=\underline{R Y}_{3}$. Hence, we have $\underline{R}_{3}=\underline{R}_{1} \subseteq \underline{R} Y_{2}$, i.e., $\underline{R} Y_{3} \subseteq \underline{R} Y_{2}$. It indicates that $Y_{3} \square_{B} Y_{2}$

Proof (n) Suppose that $Y_{1} \square_{T} Y_{2}$ and $Y_{1} \approx_{T} Y_{3}$. It implies that $\bar{R} Y_{1} \subseteq \bar{R} Y_{2}$ and $\bar{R} Y_{1}=\bar{R} Y_{3}$. Hence, we have $\bar{R} Y_{3}=$ $\bar{R} Y_{1} \subseteq \bar{R} Y_{2}$, i.e., $\bar{R} Y_{3} \subseteq \bar{R} Y_{2}$. It indicates that $Y_{3} \square_{T} Y_{2}$.

Proof $(o)$ Suppose that $Y_{1} \square Y_{2}$ and $Y_{1} \approx_{T} Y_{3}$. It implies that $Y_{1} \square_{B} Y_{2} ; Y_{1} \square_{T} Y_{2} ; Y_{1} \approx_{B} Y_{3}$ and $Y_{1} \approx_{T} Y_{3}$. Therefore, by property $(m)$ and $(n)$ 


\section{Conclusion}

In this paper, we compare the concepts of classical set with that of rough set on two universal sets. Basic properties of rough set on two universal sets like topological characteristics, measures of uncertainty, equality and inclusion of sets are expressed in terms of binary relation. It is also observed that equality and inclusion of sets can not be decided in the absolute sense, but depend on what we know about the sets. It is also clear that, all properties of rough set on two universal sets are not absolute, but are related to what we know about them. Therefore, rough set on two universal set approach could be viewed as a subjective counterpart of the classical set theory. These results obtained are important for their application in the design of knowledge bases.

\section{References}

[1] Zadeh L A. Fuzzy sets. Information and Control, 1965, 8: 338-353.

[2] Pawlak Z. Rough sets. International Journal of Computer and Information Sciences, 1982, 11: 341-356.

[3] Pawlak, Z. Rough Sets Theoretical Aspects of Reasoning about Data, Kluwer Academic Publishers, Dordrecht, The Netherlands, 1991.

[4] Molodtsov, D. Soft set theory-First results, Computers and Mathematics with Applications, 1999, 37: 19-31.

[5] Kryszkiewlcz, M. Rough set approach to incomplete information systems, Information Sciences, 1998, 112: 39-49.

[6] Lin, T. Y. Granular computing on binary relations I: Data mining and neighborhood systems, in: Rough sets in Knowledge Discovery (Skoworn, A. and Polkowski, L. Eds.), Springer-Verlag, London, 1998, 107-121.

[7] Lin, T. Y. Granular computing: Examples, intuitions and Modeling, Proceeding of the IEEE International Conference on Granular Computing, Beijing, China, 2005, 40-44.

[8] Maji, P. K., and Roy, A. R. An application of soft sets in a decision making problem, Computers and Mathematics with Applications, 2002, 44: 10771083.

[9] Pawlak, Z. Decision rules and flow networks', European Journal of Operational Research, 2004, 154 (1): 184-190.

[10] Zhong, N., and Skowron, A. A rough set based knowledge discovery process, International Journal of Applied Mathematics Computer Science, 2001, 11 (3): 603-619.
[11] Bonikowski, Z. Algebraic structure of rough sets, in: Rough sets, Fuzzy sets and Knowledge Discovery, (Ziarko, W. P. Ed.), Springer-Verlag, London, 1994, 242-247.

[12] Kondo, M. Algebraic approach to generalized rough sets, Lecturer Notes in Artificial Intelligence, 2005, 3641: 132-140.

[13] Kondo, M. On the structure of generalized rough sets, Information Sciences, 2006, 176: 589-600.

[14] Pawlak Z, Skowron A. Rough sets: some extensions. Information Sciences, Elsevier, 2007, 177 (1): 28-40.

[15] Yao, Y. Y. Constructive and algebraic methods of the theory of rough sets, Information Sciences, 1998, 109: 21-47.

[16] Zhu, W. Generalized rough sets based on relations, Information Sciences, 2007, 177 (22): 4997-5011.

[17] Lin, T. Y. Neighborhood systems and approximation in database and knowledge based systems, Proceeding of the fourth International Symposium on Methodologies of Intelligent Systems, 1989, 75-86.

[18] Zhu, W., and Wang, F. Y. On three types of covering rough sets, IEEE transactions on Knowledge and Data Engineering, 2007, 19 (8): 1131-1144.

[19] Liu, G. L. Rough sets over the Boolean algebras, Lecture Notes in Artificial Intelligence, 2005, 3641: 24-131.

[20] Pawlak, Z., and Skowron, A. Rough sets and Boolean reasoning, Information Sciences, Elsevier, 2007, 177 (1): 41-73.

[21] Liu, G. L. Generalized rough sets over fuzzy lattices, Information Sciences, 2008, 178: 16511662.

[22] Chen, D., Zhang, W., Yeung, D. and Tsang, E. C. C. Rough approximations on a complete completely distributive lattice with applications to generalized rough sets, Information Sciences, 2006 176: $1829-1848$.

[23] Dubois, D., and Prade, H. Rough fuzzy sets and fuzzy rough sets, International Journal of General System, 1990, 17: 191-208.

[24] Yao, Y. Y. Two views of the theory of rough sets in finite universes, International Journal of Approximation Reasoning, 1996, 15: 291-317.

[25] Acharjya D P, Tripathy B K. Rough sets on fuzzy approximation spaces and applications to distributed knowledge systems. International Journal of Artificial Intelligence and Soft Computing, 2008, 1 (1): 1-14.

[26] Tripathy, B. K., and Acharjya, D. P. Knowledge mining using ordering rules and rough sets on 
fuzzy approximation spaces, International Journal of Advances in Science and Technology, 2010, 1 (3): $41-50$

[27] Tripathy, B. K. Rough sets on intuitionistic fuzzy approximation spaces, Proceedings of 3rd International IEEE Conference on Intelligent Systems, London, 2006, 776-779.

[28] Acharjya D P, Ezhilarsi L. A knowledge mining model for ranking institutions using rough computing with ordering rules and formal concept analysis. International Journal of Computer Science Issues, 2011, 8 (2): 417-425.

[29] Acharjya D P, Tripathy B K. Rough sets on intuitionistic fuzzy approximation spaces and knowledge representation. International Journal of Artificial Intelligence and Computational Research, 2009, 1 (1): 29-36.

[30] Tripathy, B. K., and Acharjya, D. P. Association rule granulation using rough sets on intuitionistic fuzzy approximation spaces and granular computing, Annals. Computer Science Series, 2011, 9 (1): 125-144.

[31] Wong, S. K. M., Wang, L. S., and Yao, Y. Y. Interval structure: a framework for representing uncertain information, Proceedings of the 8th Conference on Uncertainty in Artificial Intelligence, 1993, 336-343.

[32] Liu, G. L. Rough set theory based on two universal sets and its applications, Knowledge Based Systems, 2010, 23: 110-115.

[33] Tripathy, B. K., Acharjya, D. P., and Ezhilarasi, L. Topological characterization of rough set on two universal sets and knowledge representation, Global Trends in Information Systems and Software Applications, CCIS, 2012, 270: 68-81.

[34] Tripathy, B. K., and Acharjya, D. P. Approximation of classification and measures of uncertainty in rough set on two universal sets, International Journal of Advanced Science and Technology, 2012, 40: 77 - 90.

[35] Novotny, M., and Pawlak, Z. Characterization of rough top equalities and rough bottom equalities, Bulletin Polish Academy of Science and Mathematics, 1985, 33: 91-97.

D P Acharjya received his $\mathrm{Ph} . \mathrm{D}$ in computer science from Berhampur University, India; M. Tech. degree in computer science from Utkal University, India in 2002; M. Phil. from Berhampur University, India; and M. Sc. from NIT, Rourkela, India. He has been awarded with Gold Medal in M. Sc. Currently he is an Associate Professor in the school of computing sciences and engineering, VIT University, Vellore, India. He has authored many national and international journal papers and four books; Fundamental Approach to Discrete
Mathematics, Computer Based on Mathematics, Theory of Computation; Rough Set in Knowledge Representation and Granular Computing to his credit. $\mathrm{He}$ is associated with many professional bodies CSI, ISTE, IMS, AMTI, ISIAM, OITS, IACSIT, CSTA, IEEE and IAENG. He was founder secretary of OITS Rourkela chapter. His current research interests include rough sets, formal concept analysis, knowledge representation, data mining, granular computing and business intelligence.

B. K. Tripathy a senior professor in the school of computing sciences and engineering, VIT University, at Vellore, India. He has been awarded with Gold Medals both at graduate and post graduate levels of Berhampur University, India. Also, he has been awarded with the best post graduate of the Berhampur University. He has published more than 150 technical papers in various international journals, conferences, and Springer book chapters. He is associated with many professional bodies like IEEE, ACM, IRSS, AISTC, ISTP, CSI, AMS, and IMS. He is in the editorial board of several international journals like CTA, ITTA, AMMS, IJCTE, AISS, AIT, and IJPS. Also, he is a reviewer of international journals like Mathematical Reviews, Information Sciences, Analysis of Neural Networks, Journal of Knowledge Engineering, Mathematical Communications, and Journal of Analysis. His research interest includes fuzzy sets and systems, rough sets and knowledge engineering, data clustering, granular computing and social networks. 